\title{
Maternal hyperglycemia and adverse pregnancy outcomes in Dar es Salaam, Tanzania
}

\section{Citation}

Darling, Anne Marie, Enju Liu, Said Aboud, Willy Urassa, Donna Spiegelman, and Wafaie Fawzi. 2014. "Maternal Hyperglycemia and Adverse Pregnancy Outcomes in Dar Es Salaam, Tanzania." International Journal of Gynecology \& Obstetrics 125 (1) (April): 22-27. doi:10.1016/ j.ijgo.2013.10.007.

\section{Published Version}

doi:10.1016/j.ijgo.2013.10.007

\section{Permanent link}

http://nrs.harvard.edu/urn-3:HUL.InstRepos:26967988

\section{Terms of Use}

This article was downloaded from Harvard University's DASH repository, and is made available under the terms and conditions applicable to Open Access Policy Articles, as set forth at http:// nrs.harvard.edu/urn-3:HUL.InstRepos:dash.current.terms-of-use\#OAP

\section{Share Your Story}

The Harvard community has made this article openly available.

Please share how this access benefits you. Submit a story.

\section{Accessibility}


Int J Gynaecol Obstet. 2014 April ; 125(1): 22-27. doi:10.1016/j.ijgo.2013.10.007.

\title{
Maternal hyperglycemia and adverse pregnancy outcomes in Dar es Salaam, Tanzania
}

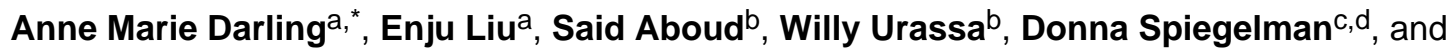 \\ Wafaie Fawzi ${ }^{a, c, e}$ \\ aDepartment of Global Health and Population, Harvard School of Public Health, Boston, USA \\ bDepartment of Microbiology and Immunology, Muhimbili University of Health and Allied \\ Sciences, Dar es Salaam, Tanzania \\ 'Department of Epidemiology, Harvard School of Public Health, Boston, USA \\ dDepartment of Biostatistics, Harvard School of Public Health, Boston, USA \\ eDepartment of Nutrition, Harvard School of Public Health, Boston, USA
}

\begin{abstract}
Objective-To evaluate maternal glucose levels during pregnancy as a predictor of adverse perinatal outcomes in Dar es Salaam, Tanzania.

Methods-Random blood glucose measurements were analyzed from 3833 pregnant women enrolled in a randomized trial to assess the impact of multivitamins on pregnancy outcomes in Dar es Salaam between August 2001 and July 2004. Information on maternal and neonatal morbidity was recorded at monthly study visits, delivery, and 6 weeks postpartum. Generalized estimating equations specified with a binomial distribution and log-link function were used to determine the relationship between elevated glucose $(>7.8 \mathrm{mmol} / \mathrm{L})$ and pregnancy outcomes.
\end{abstract}

Results-In total, 25 women had elevated glucose $(0.7 \%)$. Hyperglycemia was associated with an increased risk of delivery before 37 weeks [relative risk (RR), $2.11 ; 95 \%$ confidence interval [CI], 1.07-4.13; $P=0.03$ ), delivery before 34 weeks (RR, 4.15; 95\% CI, 1.43-12.03, $P=0.009$ ), incident gestational hypertension (RR, 2.90; 95\% CI, 1.24-6.76; $P=0.01$ ), low birth weight (RR, 2.87; 95\% CI, 1.18-6.99; $P=0.02$ ), reduced newborn head circumference (mean difference, -1.53 ; $95 \% \mathrm{CI},-2.51$ to $-0.62 ; P=0.001)$, and stillbirth (RR, 3.38; 95\% CI, $1.13-10.08 ; P=0.03)$.

Conclusion-Maternal hyperglycemia is uncommon among pregnant Tanzanian women, but nonetheless seems to increase the risk of several adverse perinatal outcomes.

() 2013 International Federation of Gynecology and Obstetrics. Published by Elsevier Ireland Ltd. All rights reserved. *Corresponding author: Anne Marie Darling, Division of Global Health and Population, Harvard School of Public Health, 677 Huntington Avenue, Boston, MA 02115, USA. Tel.: +1 617432 1222; fax: +1 617432 1355. adarling@ @ hsph.harvard.edu.

Publisher's Disclaimer: This is a PDF file of an unedited manuscript that has been accepted for publication. As a service to our customers we are providing this early version of the manuscript. The manuscript will undergo copyediting, typesetting, and review of the resulting proof before it is published in its final citable form. Please note that during the production process errors may be discovered which could affect the content, and all legal disclaimers that apply to the journal pertain.

Conflict of interest

The authors have no conflicts of interest. 


\section{Keywords}

Africa; Gestational hyperglycemia; Gestational hypertension; Low birth weight; Preterm birth; Stillbirth

\section{Introduction}

Both gestational and pre-gestational diabetes have well-known deleterious consequences for mothers and neonates, including pregnancy-induced hypertension [1,2], stillbirth [1-4], perinatal death [1,4-6], cesarean delivery [5,7], preterm delivery [1,5-8], high and low birth weight $[1,5,7]$, and both large-for-gestational age and small-for-gestational age (SGA) outcomes [6,8]. Considerable evidence also shows that milder hyperglycemia as an antecedent to overt diabetes can lead to adverse pregnancy outcomes [3,7,9-11].

The management of diabetic pregnancies may be a growing concern in sub-Saharan Africa, where diabetes has received comparatively little attention. Estimates of the prevalence of gestational diabetes in this region are scarce and have varied from $0 \%$ to $13.9 \%[1,11-13]$. Impaired glucose tolerance, a precursor to diabetes, has been reported as $0 \%$ among pregnant women in Tanzania [14,15] and 7.3\% among those in South Africa [16].

Predictions indicate that, between 2010 and 2030, sub-Saharan Africa will experience the largest percentage increase in diabetes of any region [17]. The prevalence of diabetes among adults in sub-Saharan Africa is expected to increase from 3.4\% to $4.7 \%$ during this time, which will translate to 11.8 million new cases. As a result, increasing numbers of women in this region will enter pregnancy with abnormal glucose regulation or will experience its onset during gestation and subsequently face a substantial risk of pregnancy complications.

The aim of the present study was to examine the effect of plasma glucose levels in pregnancy on adverse perinatal outcomes within a cohort of women who were previously enrolled in a randomized controlled trial of daily multivitamin supplementation (vitamins B, $\mathrm{C}$, and E) versus placebo in Dar es Salaam, Tanzania, [18].

\section{Materials and methods}

The present study retrospectively analyzed data from pregnant women who were enrolled in a double-blind, randomized, placebo-controlled trial at participating prenatal clinics in Dar es Salaam, Tanzania, between August 1, 2001, and July 31, 2004 [18]. Ethics approval for the trial was obtained from the institutional review boards of Muhimbilli University of Health and Allied Sciences in Dar es Salaam, Tanzania, and Harvard School of Public Health in Boston, MA, USA. Written informed consent was obtained from all women.

Women eligible for the original trial were HIV-negative, had a pregnancy of 12-27 weeks of gestation, and were planning to stay in Dar es Salaam for at least 1 year after delivery. The present analysis included only women who had available glucose measurements (supply limitations precluded the measurement of glucose levels for every trial participant). 
Glucose was measured in plasma drawn from participants at regularly scheduled study visits to prenatal clinics. Most (90\%) samples were obtained between 22-34 weeks of gestation. Glucose testing was performed with the Roche Hitachi 911 chemistry analyzer (Roche Diagnostics, Indianapolis, IN, USA).

Individuals with abnormal glucose levels were referred for treatment as per standard of care. Because only random plasma glucose levels were available, it was not possible to follow conventional guidelines for the diagnosis of gestational diabetes or impaired glucose tolerance. Instead, glucose levels exceeding $7.8 \mathrm{mmol} / \mathrm{L}(140 \mathrm{mg} / \mathrm{dL})$ were considered to be elevated. This cutoff value represents the typical upper threshold for peak postprandial glucose during pregnancy [19]. The follow-up period for the present analysis began at the time of glucose measurement and ended at 6 weeks postpartum.

Information on sociodemographic characteristics was collected through maternal interviews at baseline. Gestational age was determined by the physician using the date of the last menstrual period. At each monthly visit, participants underwent physical examination and anthropometric assessments. Study nurses recorded single values for systolic and diastolic blood pressure at each visit with a mercury sphygmomanometer while participants were at rest. Routine laboratory tests were conducted as described elsewhere [17].

Maternal complications included preterm delivery, severe preterm delivery, hypertension, cesarean delivery, and severe anemia. All deliveries that occurred before 37 weeks of gestation were considered preterm. Those that occurred before 34 weeks were considered severely preterm. Hypertension was defined as a systolic blood pressure of $140 \mathrm{~mm} \mathrm{Hg}$ or more or a diastolic blood pressure of $90 \mathrm{~mm} \mathrm{Hg}$ or more at any time during pregnancy. Cesarean delivery was recorded by the research midwives who attended to participants during labor.

Newborn growth parameters included birth weight, SGA status, crown-heel length, head circumference, and placental weight. After delivery, research midwives weighed neonates and placentas to the nearest $10 \mathrm{~g}$ and measured newborn length and head circumference to the nearest $0.1 \mathrm{~cm}$. Neonates weighing less than $2500 \mathrm{~g}$ were categorized as low birth weight. Those weighing more than $4000 \mathrm{~g}$ were categorized as macrosomic. SGA status was defined as a birth weight below the 10th percentile for gestational age. US standards were used as the "reference population" because they might provide a better reflection of growth potential unaffected by nutritional deprivation than local norms [17]. Fetal and perinatal mortality outcomes included fetal loss (any death occurring before delivery), stillbirth (deaths occurring at 28 gestational weeks or more), perinatal death (deaths that occurred between 28 weeks of gestation and 1 week of delivery) and early infant deaths (deaths that occurred before 6 weeks postpartum).

All statistical analyses were performed via SAS version 9.2 (SAS Institute, Cary, NC, USA). Relative risks (RRs) and 95\% confidence intervals (CIs) were determined by using generalized estimating equations specified with the log-link function for all dichotomous outcomes. In most cases, however, the log-binomial models failed to converge and were 
replaced with log-Poisson models, which provide consistent but not fully efficient estimates of the RR and its CIs [20].

For models of hypertension in pregnancy, a compound symmetry working correlation matrix was used to account for repeated measures of blood pressure [21]. Women with hypertension at baseline were excluded from the analysis of this outcome. A compound symmetry working correlation matrix was also specified for analyses of birth outcomes to account for correlations due to twinning. Continuous endpoints were assessed with generalized estimating equations with the use of the identity link and Gaussian variance function and a compound symmetry working correlation matrix. Restricted cubic splines [22] were used to model continuous glucose levels in relation to the outcomes of interest. All analyses of birth outcomes were restricted to live births.

All multivariate models included age ( $<20$ years, 20 to $<25$ years, 25 to $<30$ years, 230 years), nearest body mass index (BMI, calculated as weight in kilograms divided by the square of height in meters) measurement prior to the time of glucose measurement $(<18.5$ $\mathrm{kg} / \mathrm{m}^{2}, 18.5-24.9 \mathrm{~kg} / \mathrm{m}^{2}, 25.0-29.9 \mathrm{~kg} / \mathrm{m}^{2}, 30.0 \mathrm{~kg} / \mathrm{m}^{2}$ ), quartiles of nearest mid-upper arm circumference prior to the time of glucose measurement, Filmer-Pritchett wealth score below median (yes/no), and receipt of multivitamins (yes/no).

In addition, for each separate outcome, the following characteristics were considered to be potential confounders if they predicted the outcome in univariate analysis at a $P$ value of 0.20 or less: literacy (yes/no); marital status (yes/no); dependence on others for economic support (yes/no); low daily per capita food expenditure (yes/no); education (0-4, 5-7, 8-11, $\geq 12$ years); frequency of meat or fish intake ( $<1$ per month, 1-3 times per month, about once/week, 2-4 times per week, 5-7 times per week); gravidity $(0,1, \geq 2)$; gestational age at time of glucose measurement ( $<25$ weeks, $\geq 25$ weeks); weight gain from study entry to the time of glucose measurement ( $<3 \mathrm{~kg}, 3$ to $<6 \mathrm{~kg}, 6$ to $<9 \mathrm{~kg}, \geq 9 \mathrm{~kg}$ ); quartiles of nearest triceps skin fold thickness measurement prior to time of glucose measurement; nearest hemoglobin measurement prior to time of glucose measurement $(<8.5 \mathrm{~g} / \mathrm{L}, 8.5-10.9 \mathrm{~g} / \mathrm{L}$, $\geq 11.0 \mathrm{~g} / \mathrm{dL}$ ); smoking (yes/no); year of recruitment (2001, 2002, 2003, 2004); district of recruitment (Ilala, Temeke, Kinondoni); history of hypertension during the current pregnancy (yes/no; for analysis of hypertension); family history of hypertension (yes/no; for analysis of hypertension); family history of diabetes (yes/no); history of a low birth weight newborn (yes/no; for analyses of fetal growth) history of fetal loss (yes/no; for analyses of fetal and infant mortality); use of hematinics during this pregnancy (yes/no; for analyses of hematologic outcomes). Missing indicators were used to retain observations in the analyses for variables where more than $1 \%$ of total observations were missing [23]. Observations containing missing values for model covariates with $1 \%$ or fewer total observations missing were not retained in the analyses. A $P$ value of less than 0.05 was considered to be statistically significant.

\section{Results}

The original study population comprised 8468 pregnant women [17], of whom 3383 had available glucose measurements and were included in the present analysis. Figure 1 shows 
derivation of the study population. Women with available glucose measurements ( $\mathrm{n}=3383)$ were comparable to those without glucose measurements $(n=5045)$ with respect to baseline characteristics (Table 1).

Of the 3383 participants included in the analysis, $25(0.7 \%)$ had elevated glucose levels. These participants were similar to those with normal glucose levels in terms of baseline reproductive and socioeconomic characteristics at the time of enrollment into the larger trial (Table 2). On average, participants with elevated glucose levels tended to be older and had higher socio-economic status, higher BMI, and greater mid-upper arm circumference. A substantially higher proportion of participants with elevated glucose had received the multivitamin regimen during the trial.

Associations between maternal outcomes and glucose status are shown in Table 3. Women with elevated glucose levels had a significantly and independently increased risk of preterm delivery, severe preterm delivery, and incident gestational hypertension in multivariate analyses. Risk of cesarean delivery seemed to increase at glucose values both above and below normal levels (Figure 2; $P$ value for nonlinearity, 0.01).

Elevated glucose also significantly increased the risk of low birth weight (Table 4). The multivariate RR for low birth weight $(<2500 \mathrm{~kg}$ ) was 2.87 (95\% CI, 1.18-6.99; $P=0.02$ ). The relationship between continuous glucose levels and low birth weight deviated from linearity (multivariate $P$ value for nonlinearity, 0.03 ). When the outcome definition was narrowed to low birth weight newborns who were born preterm, the association with elevated glucose levels became stronger and remained borderline significant (multivariate RR, 3.78; 95\% CI, 1.01-14.19, $P=0.05)$. However, the increased risk for low birth weight term neonates among those with elevated glucose levels was nonsignificant $(P=0.2)$. The average head circumference of newborns of mothers with elevated glucose was $1.57 \mathrm{~cm}$ smaller than that of newborns of mothers with normal glucose levels (95\% CI, -2.51 to $0.62 ; P=0.002)$ in multivariate analysis.

Continuous glucose levels had a nonlinear association with head circumference (multivariate $P$ value for nonlinearity, 0.05$)$ and a positive association with crown-heel length (multivariate OR, $0.27 ; 95 \% \mathrm{CI}, 0.10-0.45$ ). Women with elevated glucose levels were 3.38 times (95\% CI, 1.13-10.08; $P=0.03$ ) more likely to experience fetal loss, and 3.58 (95\% CI, $1.19-10.75 ; P=0.02$ ) times more likely to experience stillbirth in particular (Table 5).

\section{Discussion}

The present results show that elevated glucose levels are rare among pregnant women in Dar es Salaam, but nonetheless seem to strongly predict several adverse pregnancy outcomes, including pre-term delivery, hypertension, low birth weight, decreased head circumference, fetal loss, and stillbirth. They also suggest that crown-heel length increases with increasing glucose levels, and the likelihood of a Cesarean delivery increases with both increasing and decreasing glucose levels. These associations persisted even after adjustment for potential confounders such as age, maternal anthropometry, socioeconomic status, and receipt of 
multivitamins, all of which differed between participants with normal and elevated glucose levels.

Two studies in the early 1990s found no evidence of impaired glucose tolerance among pregnant Tanzanian women $[14,15]$. The present results also suggest that elevated glucose levels are rare among pregnant women in Dar es Salaam, although a conservative definition of exposure was adopted because information was lacking on the timing of meal consumption prior to the glucose test. Kileo [24] observed evidence of impaired glucose tolerance among $11.1 \%$ of pregnant women who underwent random blood glucose testing at Muhimbili National Hospital, Dar es Salaam, using a less conservative definition that accounted for time since last meal. Those more recent data, which are similar to estimates from elsewhere in sub-Saharan Africa [1,11-13], suggest that gestational hyperglycemia is indeed a concern among Tanzanian pregnant women.

Direct comparison of the present findings with previous studies is limited by differences in methods of glucose assessment. Nevertheless, the results do support past research suggesting that elevated glucose levels below the threshold for overt diabetes are associated with preterm birth [9], stillbirth [3], hypertension [10], and cesarean delivery [7]. The increased risks for low birth weight and reduced head circumference observed among the study women with elevated glucose levels suggest that maternal hyperglycemia may adversely impact fetal growth in this population, although the significant increase in crown-heel length seen with increasing glucose levels seems to contradict this supposition. Taken together, these findings add to the abundance of data suggesting that glucose intolerance in pregnancy has pathophysiologic consequences even before it progresses to overt diabetes. It should be noted that these associations were noted in the present study despite the referral of hyperglycemic participants for treatment as per standard of care. Untreated women in this setting may have an even higher risk of these morbidities, given that treatment reduces the risk of pregnancy complications.

The risk of cesarean delivery unexpectedly increased with decreasing glucose levels in the present study. To our knowledge, such a finding has not previously been reported. We can only speculate that reduced availability of maternal glucose may have contributed to fetal growth restriction [25], leading to fetal distress during labor. Among the 22 participants who delivered SGA neonates by cesarean, 17 had glucose levels of $3.1 \mathrm{mmol} / \mathrm{L}$ or lower.

The use of random blood glucose levels to measure hyperglycemia instead of a more sensitive and specific test is a major limitation of the study. In addition, the low prevalence of hyperglycemia limited the precision of the present estimates of RR. Furthermore, the use of date of last menstrual period to determine gestational age may have resulted in some misclassification of preterm deliveries. Any such misclassification would have occurred independently of exposure status, however, and would lead to an underestimation of the RR. Although the assessment of a large number of outcomes also increases the potential for false-positive findings, the present outcome selection was hypothesis-driven and the results largely concur with previous research. The strengths of the present study include the prospective design and ability to control for a wide range of covariates as potential 
confounders. In addition, the similarity of participants with and without glucose measurements was confirmed in support of the external validity of the findings.

In conclusion, the study found evidence of the well-established association between abnormally elevated glucose levels and adverse pregnancy outcomes in urban Tanzania, where many of these outcomes occur disproportionately. Larger studies using internationally recommended screening procedures for gestational diabetes should confirm these findings so that improvements in prenatal care for hyperglycemic women can be made in this setting.

\section{Acknowledgments}

Financial support was provided by a grant from the National Institute of Child Health and Human Development (NICHD R01 37701)

\section{References}

1. Ozumba BC, Obi SN, Oli JM. Diabetes mellitus in pregnancy in an African population. Int J Gynecol Obstet. 2004; 84(2):114-119.

2. Keshavarz M, Cheung NW, Babaee GR, Moghadam HK, Ajami ME, Shariati M. Gestational diabetes in Iran: incidence, risk factors and pregnancy outcomes. Diabetes Res Clin Pract. 2005; 69(3):279-286. [PubMed: 16098925]

3. Wood SL, Jick H, Sauve R. The risk of stillbirth in pregnancies before and after the onset of diabetes. Diabet Med. 2003; 20(9):703-707. [PubMed: 12925047]

4. Macintosh MC, Fleming KM, Bailey JA, Doyle P, Modder J, Acolet D, et al. Perinatal mortality and congenital anomalies in babies of women with type 1 or type 2 diabetes in England, Wales, and Northern Ireland: population based study. BMJ. 2006; 333(7560):177. [PubMed: 16782722]

5. Vangen S, Stoltenberg C, Holan S, Moe N, Magnus P, Harris JR, et al. Outcome of pregnancy among immigrant women with diabetes. Diabetes Care. 2003; 26(2):327-332. [PubMed: 12547857]

6. Vääräsmäki MS, Hartikainen A, Anttila M, Pramila S, Koivisto M. Factors predicting peri- and neonatal outcome in diabetic pregnancy. Early Hum Dev. 2000; 59(1):61-70. [PubMed: 10962168]

7. Metzger BE, Lowe LP, Dyer AR, Trimble ER, Chaovarindr U, et al. HAPO Study Cooperative Research Group. Hyperglycemia and adverse pregnancy outcomes. N Engl J Med. 2008; 358(19): 1991-2002. [PubMed: 18463375]

8. Yang J, Cummings EA, O'connell C, Jangaard K. Fetal and neonatal outcomes of diabetic pregnancies. Obstet Gynecol. 2006; 108(3 Pt 1):644-650. [PubMed: 16946226]

9. Hedderson MM, Ferrara A, Sacks DA. Gestational diabetes mellitus and lesser degrees of pregnancy hyperglycemia: association with increased risk of spontaneous preterm birth. Obstet Gynecol. 2003; 102(4):850-856. [PubMed: 14551018]

10. Innes KE, Wimsatt JH, McDuffie R. Relative glucose tolerance and subsequent development of hypertension in pregnancy. Obstet Gynecol. 2001; 97(6):905-910. [PubMed: 11384694]

11. Tandu-Umba B, Mbangama Muela A. Outcome-based diagnosis of hyperglycemia in pregnancy in Kinshasa, Democratic Republic of Congo. Int J Gynecol Obstet. 2013; 120(1):93-94.

12. Hall V, Thomsen RW, Henriksen O, Lohse N. Diabetes in Sub Saharan Africa 1999-2011: epidemiology and public health implications. A systematic review. BMC Public Health. 2011; 11:564. [PubMed: 21756350]

13. Kuti MA, Abbiyesuku FM, Akinlade KS, Akinosun OM, Adedapo KS, Adeleye JO, et al. Oral glucose tolerance testing outcomes among women at high risk for gestational diabetes mellitus. J Clin Pathol. 2011; 64(8):718-721. [PubMed: 21606228]

14. Swai AB, Kitange HM, McLarty DG, Kilima PM, Masuki G, Mtinangi BL, et al. No deterioration of oral glucose tolerance during pregnancy in rural Tanzania. Diabet Med. 1991; 8(3):254-257. [PubMed: 1828741] 
15. Lutale JK, Justesen A, Swai AB, Alberti KG, McLarty DF. Glucose tolerance during and after pregnancy in nondiabetic women in an urban population in Tanzania. Diabetes Care. 1993; 16(4): 575-577. [PubMed: 8462381]

16. Mamabolo RL, Alberts M, Levitt NS, Delemarre-van de Waal HA, Steyn NP. Prevalence of gestational diabetes mellitus and the effect of weight on measures of insulin secretion and insulin resistance in third-trimester pregnant rural women residing in the Central Region of Limpopo Province, South Africa. Diabet Med. 2007; 24(3):233-239. [PubMed: 17263763]

17. Shaw JE, Sicree RA, Zimmet PZ. Global estimates of the prevalence of diabetes for 2010 and 2030. Diabetes Res Clin Pract. 2010; 87(1):4-14. [PubMed: 19896746]

18. Fawzi WW, Msamanga GI, Urassa W, Hertzmark E, Petraro P, Willett WC, et al. Vitamins and perinatal outcomes among HIV-negative women in Tanzania. N Engl J Med. 2007; 356(14):14231431. [PubMed: 17409323]

19. International Diabetes Federation. [Accessed September 18, 2013] Guideline for Management of PostMeal Glucose in Diabetes. 2011. https://www.idf.org/2011-guideline-management-postmealglucose-diabetes. Published 2011.

20. Zou G. A modified poisson regression approach to prospective studies with binary data. Am J Epidemiol. 2004; 159(7):702-706. [PubMed: 15033648]

21. Fitzmaurice, GM.; Laird, NM.; Ware, JH. Applied Longitudinal Analysis. New York, NY: Wiley; 2004.

22. Durrleman S, Simon R. Flexible regression models with cubic splines. Stat Med. 1989; 8(5):551561. [PubMed: 2657958]

23. Miettinen, OS. Theoretical Epidemiology: Principles of Occurrence Research in Medicine. New York, NY: John Wiley \& Sons; 1985.

24. Kileo, J. master's thesis. Dar es Salaam, Tanzania: Muhimbili University of Health and Allied Sciences; 2011. Prevalence of Gestational Diabetes Mellitus, Associated Risk Factors and Outcome in Pregnant Women Attending Antenatal Care at Muhimbili National Hospital.

25. Bhat RG, Bhagya KV, Kumar P. Association of low maternal plasma glucose after oral glucose challenge test with small for gestational age neonate. Int J Infertility Fetal Med. 2012; 3(1):22-25. 


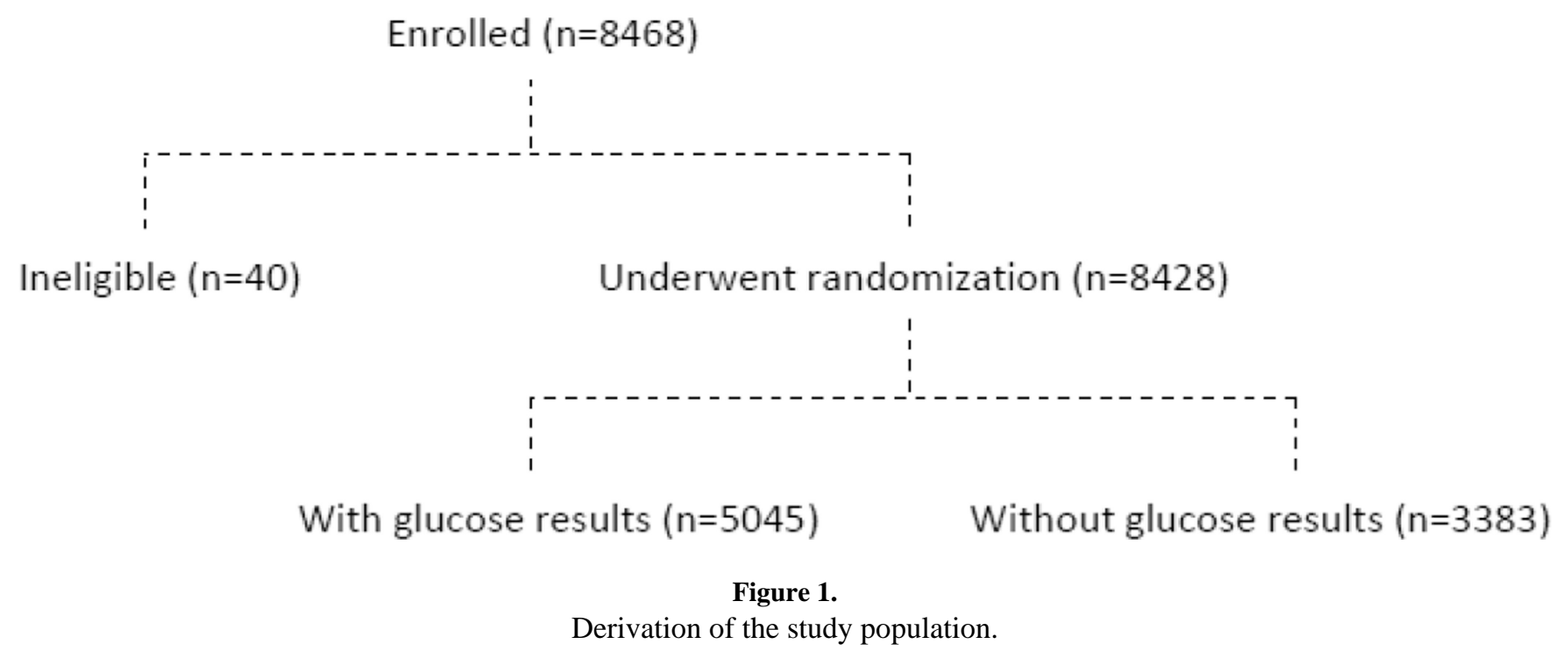

Int J Gynaecol Obstet. Author manuscript; available in PMC 2015 April 01. 


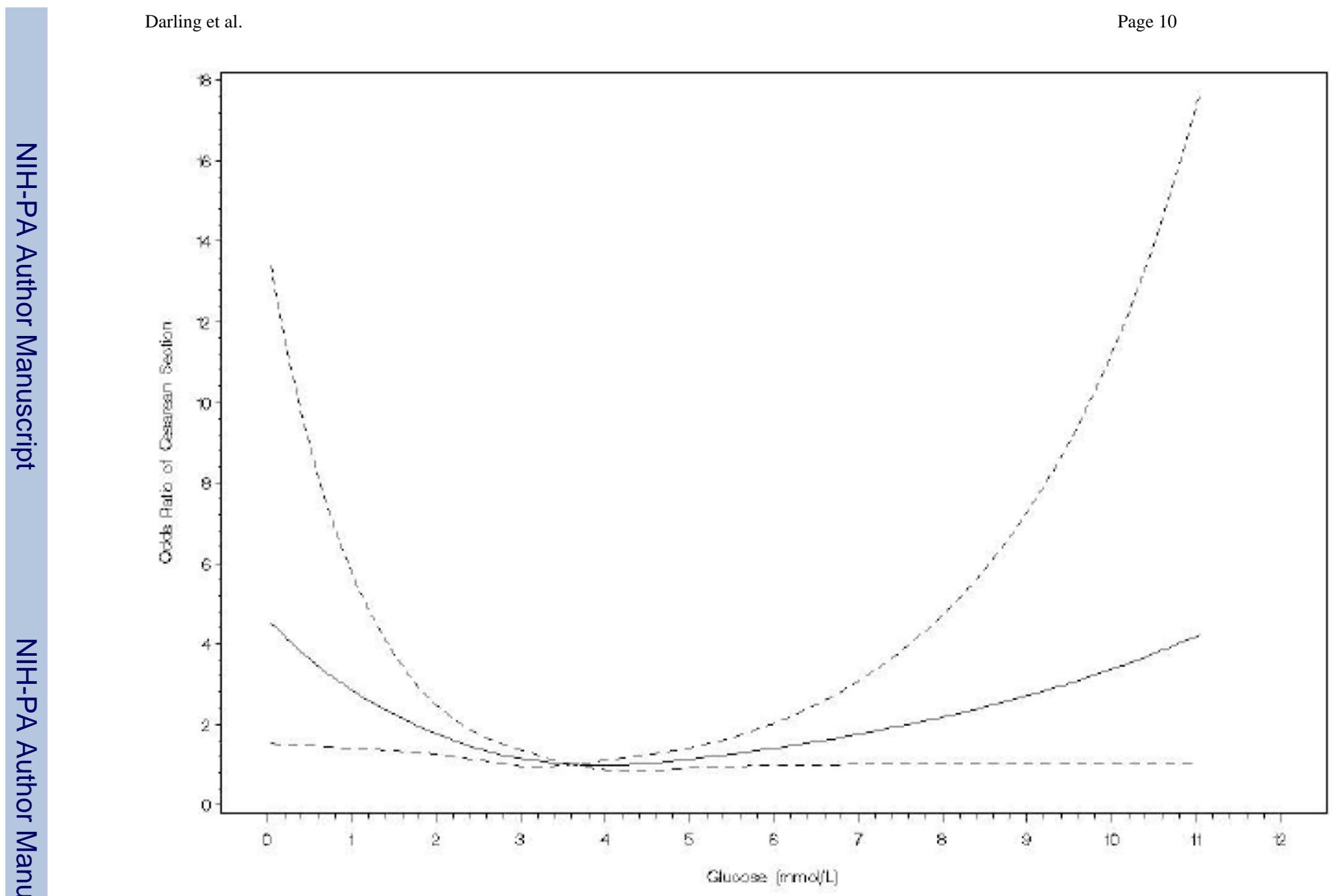

Figure 2.

Odds ratio of cesarean delivery by blood glucose levels. Broken lines represent $95 \%$ confidence intervals. 
Table 1

Comparison of baseline characteristics between women with glucose data available and those without ${ }^{a}$

\begin{tabular}{lll}
\hline Characteristic & $\begin{array}{l}\text { Glucose results available } \\
(\mathbf{n = 3 3 8 3})\end{array}$ & $\begin{array}{l}\text { Glucose results unavailable } \\
(\mathbf{n = 5 0 4 5})\end{array}$ \\
\hline Age, y & $25.2 \pm 5.0$ & $25.1 \pm 5.0$ \\
Literate & 88 & 87 \\
Completed secondary education & 5 & 5 \\
Married or cohabitating & 89 & 88 \\
Economically dependent on others & 98 & 98 \\
Low daily per capita expenditure on food & 36 & 42 \\
Filmer-Pritchett wealth score less than median & 48 & 48 \\
Nulliparous & 47 & 45 \\
Gestational age, wk & $21.5 \pm 3.3$ & $21.0 \pm 3.5$ \\
BMI & $24.5 \pm 3.6$ & $24.6 \pm 3.9$ \\
Mid-upper arm circumference, cm & $26.3 \pm 3.2$ & $26.5 \pm 3.3$ \\
Triceps skin fold thickness, mm & $18.6 \pm 6.5$ & $19.0 \pm 6.7$ \\
Received multivitamin regimen & 51 & 49 \\
\hline
\end{tabular}

Abbreviation: BMI, body mass index (calculated as weight in kilograms divided by the square of height in meters).

${ }^{a}$ Values are given as mean $\pm \mathrm{SD}$ or percentage. 
Table 2

Baseline characteristics of women enrolled in the trial with available glucose levels $(n=3383)$

\begin{tabular}{lll}
\hline Characteristic & $\begin{array}{l}\text { Normal glucose levels } \\
(\mathbf{n = 3 3 5 8})\end{array}$ & $\begin{array}{l}\text { Hyperglycemia or diabetes } \\
\text { (glucose }>\text { 7.8 mmol/L) } \\
(\mathbf{n = 2 5})\end{array}$ \\
\hline Age, y & $25.2 \pm 5.1$ & $26.65 \pm 5.58$ \\
Literate & 88 & 92 \\
Married or cohabitating & 89 & 100 \\
Completed secondary education & 5 & 2 \\
Economically dependent on others & 98 & 100 \\
Low daily per capita expenditure on food & 36 & 42 \\
Filmer-Pritchett wealth score less than median & 48 & 21 \\
Nulliparous & 47 & 58 \\
Gestational age, wk & $27.6 \pm 3.8$ & $27.1 \pm 1.8$ \\
BMI & $25.4 \pm 3.8$ & $27.8 \pm 2.3$ \\
Mid-upper arm circumference, cm & $26.4 \pm 5.0$ & $27.5 \pm 1.5$ \\
Triceps skin fold thickness, mm & $18.3 \pm 6.5$ & $18.2 \pm 2.6$ \\
Received multivitamin regimen & 51 & 34 \\
\hline
\end{tabular}

Abbreviation: BMI, body mass index (calculated as weight in kilograms divided by the square of height in meters).

${ }^{a}$ Values are given as mean $\pm \mathrm{SD}$ or percentage. 


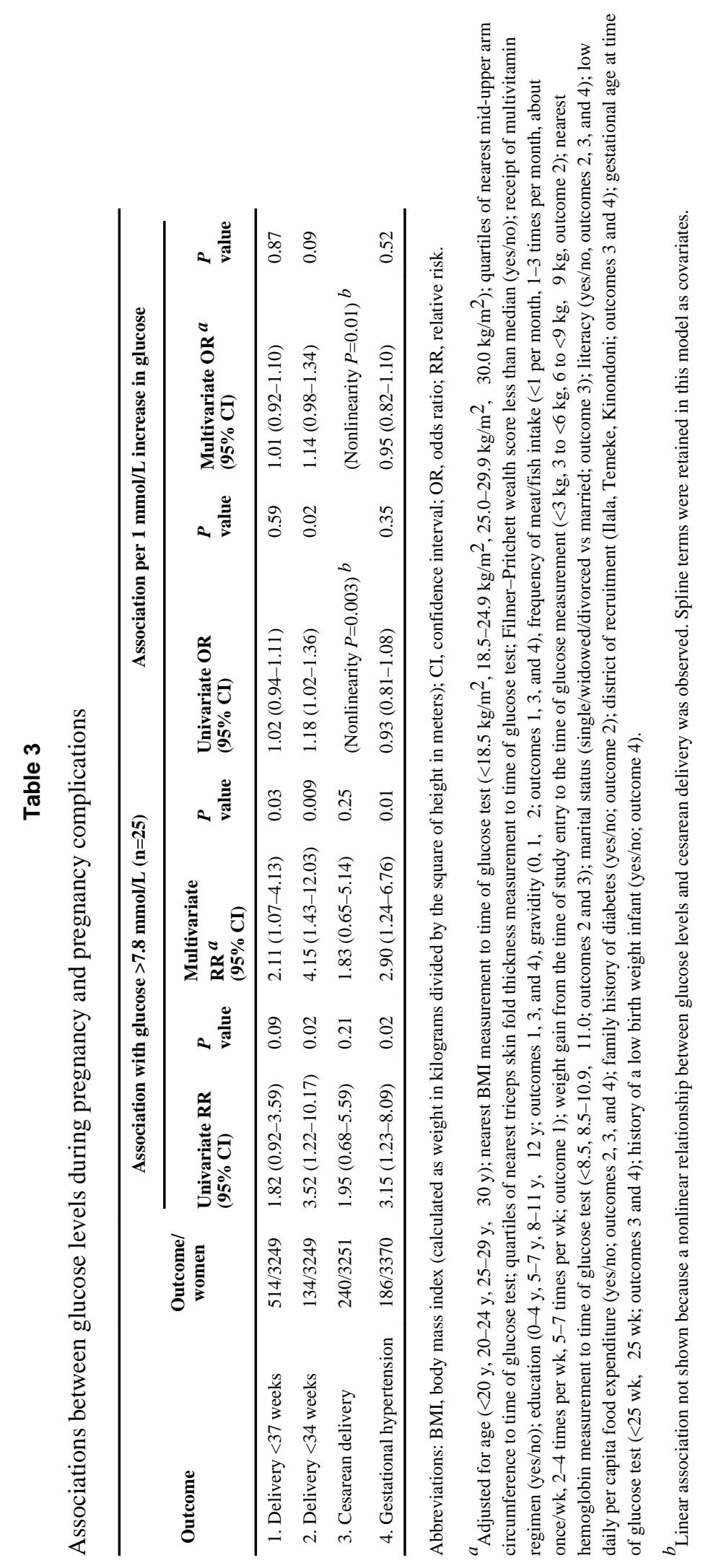

Int J Gynaecol Obstet. Author manuscript; available in PMC 2015 April 01. 


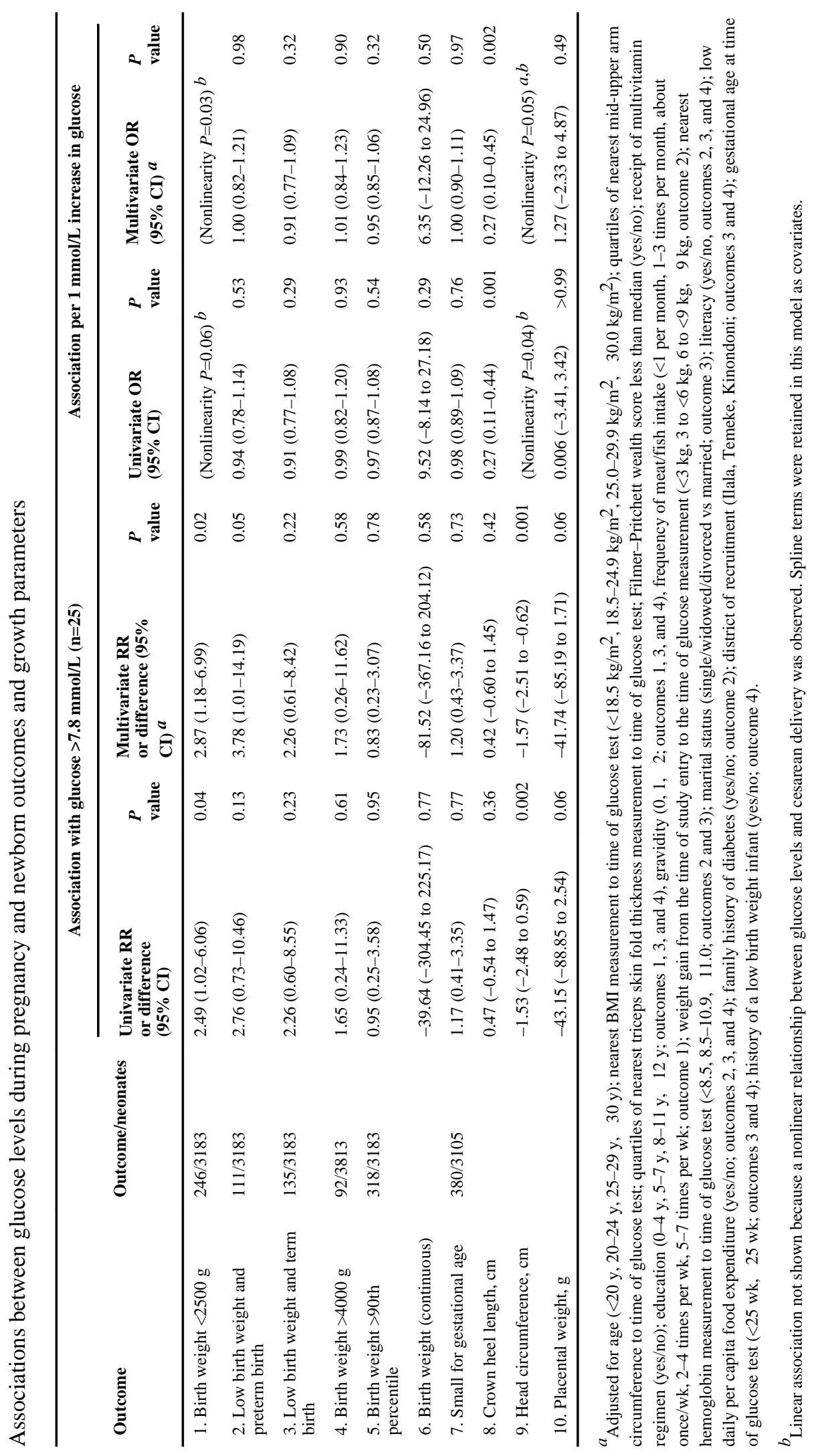




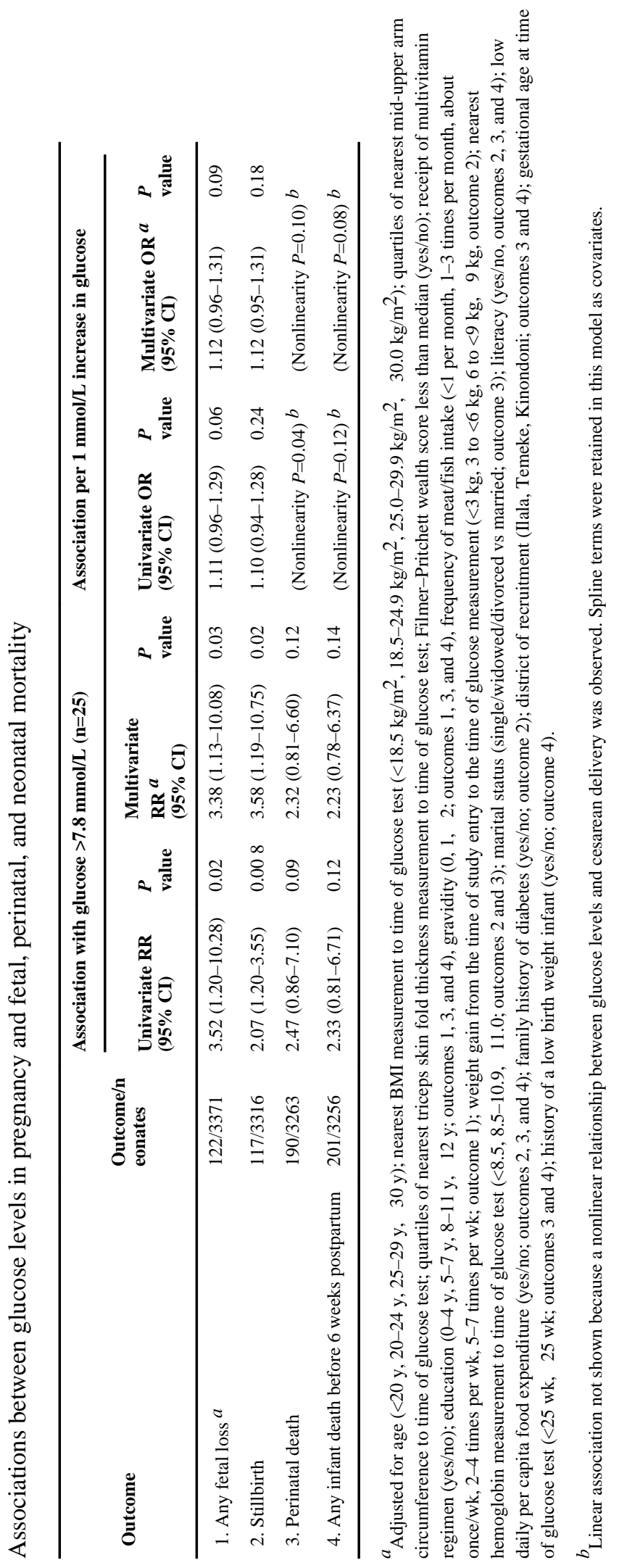

